\title{
Preliminary guidelines for diagnosing and treating tuberculosis in patients with rheumatoid arthritis in immunosuppressive trials or being treated with biological agents
}

\author{
D E Furst, J Cush, S Kaufmann, J Siegel, R Kurth
}

Ann Rheum Dis 2002;61 (Suppl II):ii62-ii63

\section{ACTIVE TUBERCULOSIS \\ Definition}

Clinically suspicious circumstances associated with bacteriological positivity, using standard techniques and including resistance testing when feasible. Note: This does not apply to children, where AFB culture may be negative.

Action: 4 Drug treatment

Class A Evidence

\section{LATENT TUBERCULOSIS \\ Definition}

Requires both " 1 " and " 2 ":

1 No signs or symptoms but in the presence of any of the following risk factors:

(a) HIV infection

(b) Previous close tuberculosis contact

(c) Ghon complex or other typical chest radiographic findings. The significance of scarring is unknown but is dependent on concomitant clinical signs and/or symptoms.

(d) Immunosuppressed host or patient on immunosuppressive therapy

(e) Underlying disease that predisposes to tuberculosis reactivation such as diabetes, cirrhosis, or alcoholism

2 A positive PPD-(intermediate strength, irrespective of past BCG use). "For persons with HIV infection, who are receiving immunosuppressive therapy, who have had recent close contact with persons with infectious tuberculosis or who have abnormal chest radiographs consistent with prior tuberculosis, $5 \mathrm{~mm}$ of induration is considered positive. For other persons with an increased probability of recent infection or with other clinical conditions that increase the risk for progression to active tuberculosis, $>10 \mathrm{~mm}$ of induration is considered positive. These include recent immigrants from high prevalence countries, injection drug users, residents and employees of high risk conquered settings (for example, healthcare workers with exposure tuberculosis) mycobacterial and the laboratory personnel, persons with clinical conditions such as silicosis, diabetes mellitus, chronic renal failure, leukaemia and lymphoma, cancer of the head and neck or lungs, weight loss $>10 \%$ of ideal body weight, gastrectomy and digital bypass, children younger than 4 years of age or adolescents exposed to adults in high risk categories. For persons at low risk for tuberculosis, for whom tuberculin testing is not generally indicated, $>15 \mathrm{~mm}$ induration is considered positive."1

It is important to consider the implications of a negative $\mathrm{PPD}$, if one is not sure it is done in a valid matter. One option is to repeat the PPD at the same strength on the other arm within five weeks, although the strength of the repeat PPD test that is most appropriate in the presence of the immediate previous PPD is not yet known.
Another option is anergy testing. Anergy testing is controversial. It is felt that there is a low risk of tuberculosis in the presence of a negative PPD even if the patient is anergic (occurs in $0 \%$ to $10 \%$ of immunocompromised patients) because there are methodological problems in administering skin tests, as well as problems with standardisation of reagents. Furthermore, the benefits of knowing whether or not there is anergy are unknown. If it is decided to apply an "anergy panel", this should consist of a mumps and a Candida skin test or antigens for which the patient has a vaccination history, with positivity defined as $5 \mathrm{~mm}$ or more of induration.

In some areas, especially in endemic regions, a chest film, in addition, is required. If the chest film reveals a Ghon complex, it is considered positive for latent tuberculosis. The Ghon complex in the presence of enlarged mediastinal lymph nodes is usually indicative of active, primary tuberculosis. If scarring is seen, its significance is unknown but is dependent upon clinical suspicion after an appropriate history and physical examination.

PPD positive patients, should be assessed for active disease with chest radiograph plus follow up of signs and symptoms if living in or having previously lived in endemic regions. If no history of exposure in endemic regions and if the patient is not at risk (for example, HIV positivity, poor socioeconomic circumstances), no follow up chest radiograph is necessary in the absence of suspicious signs or symptoms, or both.

Sputum exaiminations are indicated in those with suspicious signs or symptoms or those with an abnormal chest radiograph.

\section{Action}

(1) If the PPD is negative, assuming a valid test, no further treatment is required.

(2) If the PPD is positive in the presence of appropriate clinical suspicion, signs or symptoms, the treatment is as follows (unless previously treated appropriately for active tuberculosis):

Usually single drug therapy (INH may be used for nine months or, alternatively, oral rifampin may be used for four months) unless the patient is from an area where resistance is or was $>10 \%$ of background. Drug therapy using two or even up to four drugs for two to three months should be used if the suspicion of resistant organisms is high.

Class A Evidence ${ }^{12}$

(3) If the PPD is negative but the chest radiograph reveals scarring or a Ghon-like complex, monitoring for histoplasmosis or other opportunistic infections is appropriate.

(4) Liver function tests should be followed up every two to four weeks while receiving antituberculous treatment and clinical symptoms or signs of toxicity (for example, nausea, vomiting, jaundice) should also be sought. 


\section{Authors' affiliations}

D E Furst, J Siegel, R Kurth, UCLA Medical School, Los Angeles, USA

J Cush, Presbyterian Hospital, Dallas, USA

S Kaufmann, R Kurth, Koch Institute, Berlin, Germany

J Siegel, FDA, Washington DC, USA

Correspondence to: Dr D E Furst, 1000 Veteran Avenue Rehab Center, Rn 32-59, Los Angeles, CA 90095, USA; defurst@mednet.ucla.edu

\section{REFERENCES}

1 Joint Statement of the ATS, CDC and Endorsed by the Council of the Infectious Disease Society of America. Targeted tuberculin testing and treatment of latent tuberculosis infection. Am J Resp Crit Care Med. 2000;161:s221-47.

2 Comstock GW. How much isoniazid is needed for prevention of tuberculosis among immunocompetent adults? International Journal of Tuberculosis and Lung Disease 1999;3:847-50

\section{Want full text but don't have a subscription?}

\section{Pay per view}

For just $\$ 8$ you can purchase the full text of individual articles using our secure online ordering service. You will have access to the full text of the relevant article for 48 hours during which time you may download and print the pdf file for personal use.

\section{www.annrheumdis.com}

